\title{
R-spondin 2 mediates neutrophil egress into the alveolar space through increased lung permeability
}

\author{
S. R. Jackson', M. F. D. M. Costa', C. F. Pastore', G. Zhao' ', A. I. Weiner', S. Adams', G. Palashikar ${ }^{1}$, K. Quansah', \\ K. Hankenson ${ }^{3}$, D. R. Herbert ${ }^{1}$ and A. E. Vaughan ${ }^{1,2^{*}}$ (1)
}

\begin{abstract}
Objective: R-spondin 2 (RSPO2) is required for lung morphogenesis, activates Wnt signaling, and is upregulated in idiopathic lung fibrosis. Our objective was to investigate whether RSPO2 is similarly important in homeostasis of the adult lung. While investigating the characteristics of bronchoalveolar lavage in $\mathrm{RSPO}$-deficient $\left(\mathrm{RSPO}^{-1-}\right.$ ) mice, we observed unexpected changes in neutrophil homeostasis and vascular permeability when compared to control $\left(\mathrm{RSPO}^{+/+}\right)$mice at baseline. Here we quantify these observations to explore how tonic RSPO2 expression impacts lung homeostasis.

Results: Quantitative PCR ( $\mathrm{PPCR}$ ) analysis demonstrated significantly elevated myeloperoxidase (MPO) expression in bronchoalveolar lavage fluid (BALF) cells from $\mathrm{RSPO}^{-1-}$ mice. Likewise, immunocytochemical (ICC) analysis demonstrated significantly more MPO+ cells in BALF from $\mathrm{RSPO}^{-1-}$ mice compared to controls, confirming the increase of infiltrated neutrophils. We then assessed lung permeability/barrier disruption via Fluorescein Isothiocyanate (FITC)dextran instillation and found a significantly higher dextran concentration in the plasma of $\mathrm{RSPO}^{-/}$mice compared to identically treated $\mathrm{RSPO}^{+/+}$mice. These data demonstrate that RSPO2 may be crucial for blood-gas barrier integrity and can limit neutrophil migration from circulation into alveolar spaces associated with increased lung permeability and/or barrier disruption. This study indicates that additional research is needed to evaluate RSPO2 in scenarios characterized by pulmonary edema or neutrophilia.
\end{abstract}

Keywords: R-spondin 2, Neutrophil migration, Lung permeability, Lung endothelial barrier, BALF, Wnt signaling, FITCdextran, Alveolar space

\section{Introduction}

R-spondins (RSPO1-4) are a family of four secreted potentiators of the canonical Wnt/ $\beta$-catenin signaling pathway, and they act as ligands for leucine-rich repeatcontaining G-protein coupled receptors (LGRs) 4-6 [1]. RSPO2 is required for normal lung morphogenesis

\footnotetext{
*Correspondence: andrewva@vet.upenn.edu

${ }^{1}$ Department of Biomedical Sciences, School of Veterinary Medicine, University of Pennsylvania, 3800 Spruce St., Old Vet 372E, Philadelphia, PA 19104, USA

Full list of author information is available at the end of the article
}

[2]. Disruption of the RSPO2 locus results in severely hypoplastic lungs at birth that exhibit more than a $50 \%$ reduction in weight [2]. Notably, the overall structures and relative ratios of differentiated pulmonary epithelial cells remain unchanged, indicating that hypoplasia is not reflective of failed differentiation, but rather reduced proliferation [2]. RSPO2 expression is largely restricted to the lung mesenchyme, suggesting a paracrine effect on the developing epithelium [2]. The physiological relevance of RSPO2 expression in adult lungs is unclear, although RSPO2 has been demonstrated to play a role in idiopathic lung fibrosis and can be used as a biomarker

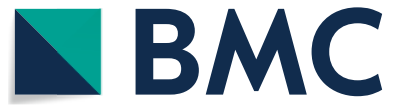

(c) The Author(s) 2020. This article is licensed under a Creative Commons Attribution 4.0 International License, which permits use, sharing, adaptation, distribution and reproduction in any medium or format, as long as you give appropriate credit to the original author(s) and the source, provide a link to the Creative Commons licence, and indicate if changes were made. The images or other third party material in this article are included in the article's Creative Commons licence, unless indicated otherwise in a credit line to the material. If material is not included in the article's Creative Commons licence and your intended use is not permitted by statutory regulation or exceeds the permitted use, you will need to obtain permission directly from the copyright holder. To view a copy of this licence, visit http://creativeco mmons.org/licenses/by/4.0/. The Creative Commons Public Domain Dedication waiver (http://creativecommons.org/publicdomain/ zero/1.0/) applies to the data made available in this article, unless otherwise stated in a credit line to the data. 
for lung cancer $[3,4]$. Whether RSPO2 plays any role in an inflammatory context (i.e., in leukocyte homeostasis or plasma extravasation) has not been investigated.

Neutrophils are bone marrow-derived polymorphonuclear leukocytes, present in the systemic circulation, that respond to inflammation, including sterile injuries (e.g. hypoxia/reperfusion) and invading pathogens [5-7]. While neutrophils play a significant role as immediate responders, their recruitment and activation are highly regulated to protect tissues from unintended effects [8]. This is particularly true in the lung microenvironment, where damage to the endothelium or epithelium from pathogenic or sterile injury can result in neutrophil migration into the alveolar space [8]. Excessive damage to alveolar structures can result in edema, gas-exchange compromise and death $[9,10]$. The loosening of endothelial cell junctions and the migration of inflammatory cells-including neutrophils-across the damaged endothelial barrier plays a key role in the pathophysiology of diseases such as acute respiratory distress syndrome (ARDS), cancer, and other inflammatory pathologies $[11,12]$. Although the canonical mechanisms governing diapedesis and leukocyte trafficking have been well described, mechanistic variations dependent on the organ milieu and inflammatory status can occur [12].

While our initial goal was to investigate a role for RSPO2 in lung repair, we observed that RSPO2 deletion in the adult lung results in aberrant neutrophil accumulation in the luminal space without a deliberate injury. Recognizing that neutrophils are normally restricted to blood vessels and that endothelial stress/damage can activate and recruit neutrophils into the interstitium or the alveolar parenchyma [13], we found that $\mathrm{RSPO}^{-1-}$ mice had significant lung barrier dysfunction. This work uncovers an important and previously unrecognized role for RSPO2 in the adult lung in regulation of neutrophil homeostasis and lung endothelial barrier maintenance.

\section{Main text \\ Methods \\ Animals and treatment}

For all experiments, mice between 6 to 8 weeks of age and $17 \mathrm{~g}$ to $20 \mathrm{~g}$ were used including males and females in equal proportions. The following strains were utilized: Inducible Cre (UBC-CreERT2) [14] (The Jackson Laboratory Stock \#007001), RSPO2 flox (a gift from Dr. Kurt Hankenson, University of Michigan), and C57BL/6 mice. No statistical method was used to predetermine sample size in any of the animal studies. The experiments were not randomized, and the investigators were not blinded to allocation during the experiments and outcome assessments.

\section{Cre recombination in vivo}

Mice were injected intraperitoneally with tamoxifen (TM) in $100 \mu \mathrm{l}$ of Mazola ${ }^{\circledR}$ corn oil ( $1 \mathrm{mg} / \mathrm{g}$ body wt) once per day, every other day for a total of 3 doses. For all analyses, tissues were collected 2 days after the last TM injection.

\section{Animal euthanasia}

Mice were placed into a closed chamber and exposed to isoflurane (Midwest Veterinary Supply) applied to compacted cotton balls until roughly 1 min after breathing stopped, followed by cervical dislocation, as approved by IACUC.

\section{Fibroblast isolation}

Fibroblasts were isolated according to our general lung cell isolation protocol previously described [15] with slight modifications: $1 \times 10^{7}$ disaggregated lung cells were plated into a Corning ${ }^{\circledR}$ 6-well clear polystyrene flat-bottom microplate (Millipore Sigma) in $\mathrm{DMEM}+20 \% \mathrm{CC}+\mathrm{P} / \mathrm{S}$ and grown in a $37{ }^{\circ} \mathrm{C}$ incubator for 9 days without passaging with media changes on days 3 and 6 before harvesting cells for mRNA or ICC analysis. For Cre-induced recombination in cultured fibroblasts, cells were treated with $(4 \mu \mathrm{M})$ of 4-Hydroxytamoxifen (4-OHT) dissolved in dimethyl sulfoxide (DMSO; Santa Cruz Biotechnology), once per day, every other day for 5 days. For qPCR and ICC analyses, fibroblast samples were collected 2 days after the last 4-OHT treatment.

\section{BALF collection}

BALF was collected according to the protocol previously described [16], followed by cytospin preparation.

\section{Cytospins}

For both cultured fibroblasts and BALF, cells were centrifuged at $570 \times g$ for $5 \mathrm{~min}$, followed by aspiration of supernatant, and cell pellets were resuspended in $1 \mathrm{ml}$ of PBS solution and fixed onto slides (Fisherbrand ${ }^{\mathrm{TM}}$ ) at $570 \mathrm{rpm}$ for $4 \mathrm{~min}$ on a Cytospin 2 (Shandon).

\section{Antibody staining}

Immunostaining was performed as previously described [16]. The following primary antibodies were used: goat anti-myeloperoxidase (MPO) (1:200 dilution; R\&D Systems), rabbit anti-RSPO2 (1:200 dilution; Proteintech). The following secondary antibodies were used: Alexa Fluor 488-conjugated donkey anti-goat (1:1000, Thermo 
Fisher Scientific), Alexa Fluor 568-conjugated donkey anti-rabbit (1:1000, Thermo Fisher Scientific).

\section{Quantification of immunostaining}

Mosaic images of cytospins were generated from multiple $20 \mathrm{X}$ fields on an upright fluorescence microscope (Leica DMi8) and tiled in LAS X software. The number of cells staining positive for the relevant antibody was manually counted and calculated as a fraction of total DAPI + cells. We quantified at least three fields per slide, each containing $\geq 300$ individual cells.

\section{Quantitative PCR ( $q P C R$ ) analysis}

RNA was isolated using the RNeasy ${ }^{\mathrm{TM}}$ (Qiagen) kit. mRNA was revered transcribed into cDNA using iScript $^{\mathrm{TM}}$ Reverse Transcription Supermix (BioRad). Total RNA input for cDNA synthesis was standardized within each experiment to the RNA isolate with the lowest concentration as measured by Nanodrop (Thermo Fisher Scientific). RT-PCR reactions were performed using SsoAdvanced ${ }^{\mathrm{TM}}$ Universal SYBR $^{\circledR}$ Green Supermix (Biorad) and run on an Applied Biosystems QuantStudio 6 Real-Time PCR System (Thermo Fisher Scientific).

\section{FITC-dextran permeability assay}

The permeability assay was performed as described in the literature $[17,18]$. Mice were anaesthetized with isoflurane and administered $40 \mu \mathrm{l}$ FITC-dextran $(10 \mathrm{mg} / \mathrm{kg}$ body weight) intranasally. After a 30-min wait to allow FITC-dextran to circulate in the blood, blood was collected via cardiac puncture, and fluorescence intensity was determined using a spectrophotometer (BioTek).

\section{Statistical analysis}

All statistical calculations were performed using GraphPad Prism. Mann-Whitney test was used to determine significance. A P value of less than 0.05 was considered significant.

\section{$P C R$ and $q P C R$ primers}

Genotyping primers Rspo2-floxA-Forward: ACTCTT ACTGCCTGGGATCCTCATT

Rspo2-floxB-Reverse: CTTCTTCTGAGCACCATC TGC

qPCR primers GAPDH Forward: AGGTCGGTGTGA ACGGATTTG

GAPDH Reverse: TGTAGACCATGTAGTTGA GGTCA

RPL37 Forward: CTCGGAGGTTACGGGACTC RPL37 Reverse: CTTGCCCTCGTAGGTAATGGG RPL19 Forward: ATG TAT CAC AGC CTG TAC CTG RPL19 Reverse: TTC TTG GTC TCT TCC TCC TTG
MPO Forward: AGTTGTGCTGAGCTGTATGGA MPO Reverse: CGGCTGCTTGAAGTAAAACAGG RSPO2 Forward: AGACGCAATAAGCGAGGTGG RSPO2 Reverse: CTGCATCGTGCACATCTGTT

\section{Results \\ Infiltration of neutrophils into bronchoalveolar lavage fluid following RSPO2 deletion}

Given that tissue repair often recapitulates features of embryonic development, where RSPO2 is critical, we generated UBC-CreERT2/RSPO2 $2^{\text {flox/flox }}$ mice to pursue the hypothesis that RSPO2 deletion would impact lung regeneration. We first confirmed successful recombination of the RSPO2 allele in adult mice after TM treatment (Fig. 1a). Additionally, we isolated lung fibroblasts from these animals, treated with 4-OHT in vitro to induce recombination, and confirmed reduction in RSPO2 transcript via $\mathrm{qPCR}$ and immunostaining (Fig. 1b-d). Before the originally planned injury experiments were initiated, we examined the lavage fluid of RSPO2 deleted mice to ensure normal levels of immune cells as determined by cytospin cell analysis. Unexpectedly, we observed MPOexpressing cells, a definitive neutrophil marker [19], in the BALF of $\mathrm{RSPO}^{-1-}$ mice at a significantly higher percentage compared to RSPO2 $2^{+/+}$mice (Fig. 2a, b). qPCR analysis demonstrated significantly higher $\mathrm{MPO}$ expression in BALF cells in $\mathrm{RSPO} 2^{-1-}$ mice compared to $\mathrm{RSPO} 2^{+/+}$mice (Fig. 2c), confirming the increase of infiltrated neutrophils. This indicates $\mathrm{RSPO} 2^{-1-}$ mice exhibit elevated neutrophil egress into the alveolar space compared to $\mathrm{RSPO} 2^{+/+}$mice in terms of both increased MPO-expressing cells and higher MPO mRNA expression in BALF cells.

\section{RSPO2 deletion increases lung barrier permeability}

Because neutrophils must exit circulation through the vasculature before translocation into the alveolar lumen [13], we hypothesized that RSPO2 deletion might induce endothelial disruption to facilitate the observed egress of neutrophils into the bronchoalveolar space. To assess lung permeability resulting from endothelial disruption, we administered FITC-dextran via intranasal instillation [16-18] and measured fluorescence in blood plasma after $30 \mathrm{~min}$. A significant increase in plasma dextran concentration was observed in $\mathrm{RSPO} 2^{-1-}$ mice compared to identically treated $\mathrm{RSPO} 2^{+/+}$mice (Fig. 3a, b). Taken together, these data indicate that RSPO2 deletion increases lung barrier permeability.

\section{Discussion}

While RSPO2 expression in the embryonic lung mesenchyme is essential for normal lung development [2], whether RSPO2 expression in the adult lung is relevant 


\section{a}
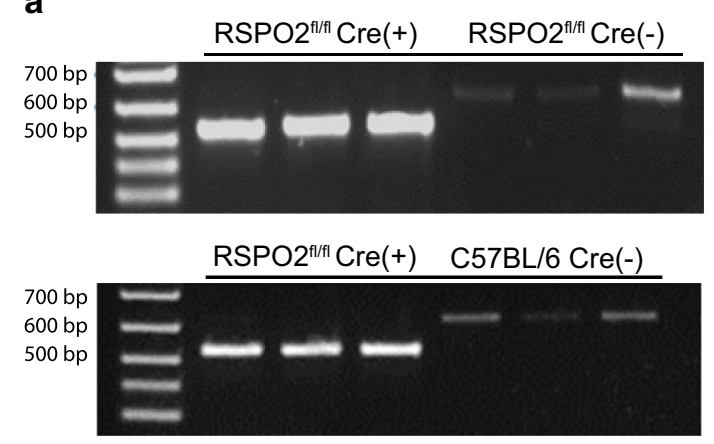

b

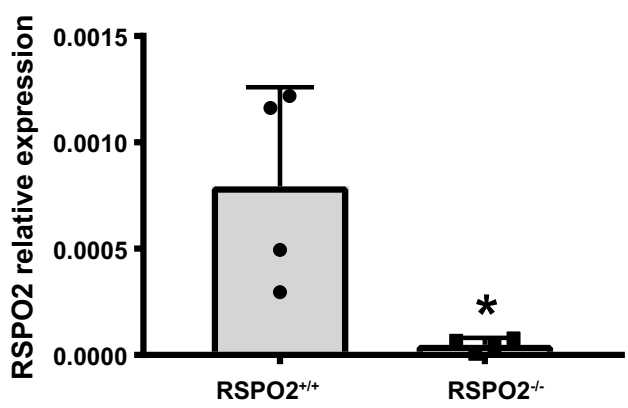

d

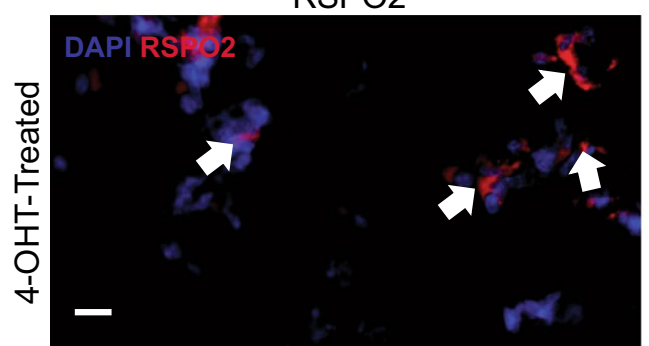

C

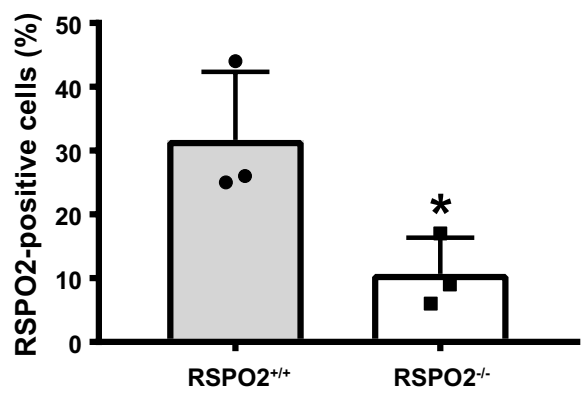

$\mathrm{RSPO}^{-1-}$

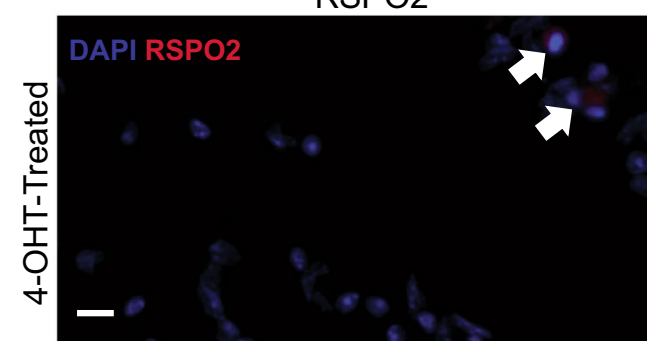

Fig. 1 Validation of RSPO2 deletion. a RSPO2 gene expression in lung homogenates from RSPO2fl/fl;UBC-Cre-ERT2(+), RSPO2fl/fl;UBC-Cre-ERT2(-), and C57BL/6 mice $48 \mathrm{~h}$ post-TM treatment. Cre-recombination of the loxP sites yields a $512 \mathrm{bp}$ fragment, whereas the wild type allele yields a non-specific 600 bp fragment. $\mathbf{b}$ qPCR analysis of RSPO2 expression in the cultured fibroblasts isolated from the lungs of RSPO2 $2^{-/-}$and RSPO2 $2^{+/+}$ mice. c, d Quantification of immunocytochemical evidence of RSPO2 expression in fibroblasts isolated from $\mathrm{RSPO}^{-/-}$and $\mathrm{RSPO}^{+/+}$mice. An arrow indicates examples of DAPI/RSPO2-double stained cells. Representative images are shown from RSPO2 ${ }^{+/+}$mice $(n=3)$ and RSPO2 ${ }^{-/-}$mice $(n=3)$ samples. ${ }^{*}=$ A P value of less than 0.05 was considered significant

in tissue homeostasis or repair has not been investigated. Our studies indicate an unexpected and biologically important role for RSPO2 in the lung as a regulator of neutrophil homeostasis and endothelial barrier function. Deletion of RSPO2 induces vascular leak and neutrophil accumulation in the airspace, indicating a novel role for $\mathrm{R}$-Spondin signaling in these contexts.

To the best of our knowledge, RSPO2 has not been previously implicated in neutrophil homeostasis/chemotaxis. Given the well described role of R-Spondins in potentiating Wnt signaling, we presume dysregulation of Wnt is the likely driver behind this phenotype. For example, Wnt5a is known to activate noncanonical Wnt pathways and activate neutrophil chemotaxis [20], and although involvement of R-spondins has not been investigated, our work supports their possible involvement.

RSPO2's role in regulation of vascular permeability is not entirely without precedent. In the adult gastrointestinal tract, another RSPO family member, RSPO3, tightens endothelial cell junctions, limiting fluid egress from 

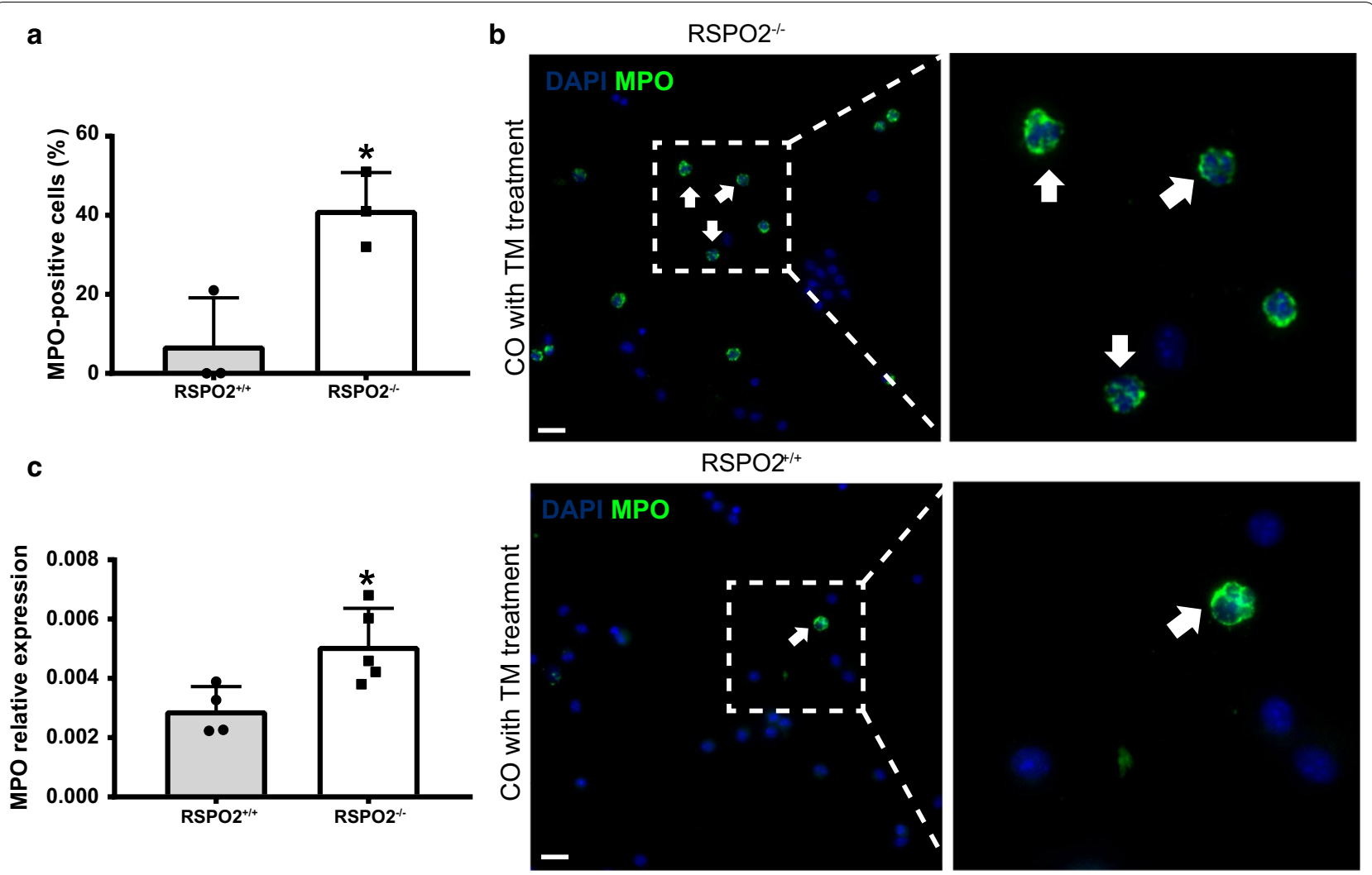

Fig. 2 RSPO2 deficiency promotes neutrophil accumulation. $\mathbf{a}$, b Quantification of ICC of MPO + cells in the BALF of RSPO2 ${ }^{+/+}$mice and RSPO2 $^{-/}$ mice $48 \mathrm{~h}$ after TM administration. An arrow indicates examples of DAPI/MPO-double stained cells. $\mathbf{c}$ GPCR analysis demonstrating a similar increase in MPO transcript in cells present in BALF of RSPO2 ${ }^{-/-}$mice. Representative images are shown from RSPO2 ${ }^{+/+}$mice $(n=3)$ and $\mathrm{RSPO}^{-/-}$mice $(n=3)$ samples. ${ }^{*}=$ A P value of less than 0.05 was considered significant

the circulation [21]. Given high expression of RSPO2 in the developing lung, RSPO2 may also play a role in the dynamic regulation of microvascular permeability that occurs at birth during the transition to air breathing [22, 23]. It is worth considering whether RSPO2, by increasing barrier integrity, may act to counterbalance other factors which decrease integrity/induce permeability, akin to how endothelin and nitric oxide act as natural counterparts to regulate vasoconstriction and vasodilation, respectively [24].

While these studies highlight potentially important new roles for RSPO2, there are many outstanding questions which require further study. First, we presume that the combination of barrier dysfunction and a second mechanism, likely involving neutrophil chemokine dysregulation, explains the appearance of neutrophils in BALF as opposed to nonspecific accumulation of circulating immune cells. Further studies are also needed to understand whether neutrophils are being actively recruited or whether they arrive in the alveolar space passively. Moreover, because we employed a broadly expressed Cre driver, whether the phenotypes described here are cell autonomous or non-autonomous is unknown. Based on developmental studies [2, 25 ] we presume the lung mesenchyme is the predominant source of RSPO2 which acts primarily in a paracrine fashion (i.e. from mesenchymal cells to endothelial cells and hematopoietic cells), but this should be formally investigated. It is also possible that autocrine RSPO2 deletion in the endothelium itself leads to the vascular phenotype. Likewise, RSPO2 deletion in the neutrophils themselves could cause spurious activation. Our findings here indicate careful, cell type-specific studies should be performed to elucidate the range of RSPO2 functions in the adult lung and beyond.

Further investigations at the molecular level will be necessary to shed more light on the exact molecular mechanisms by which RSPO2 may regulate neutrophil migration, chemoattractant responsiveness, and basal lung barrier function. Ultimately, these initial findings should seed larger efforts to elucidate specific roles for RSPO2 in lung homeostasis and disease. 
a

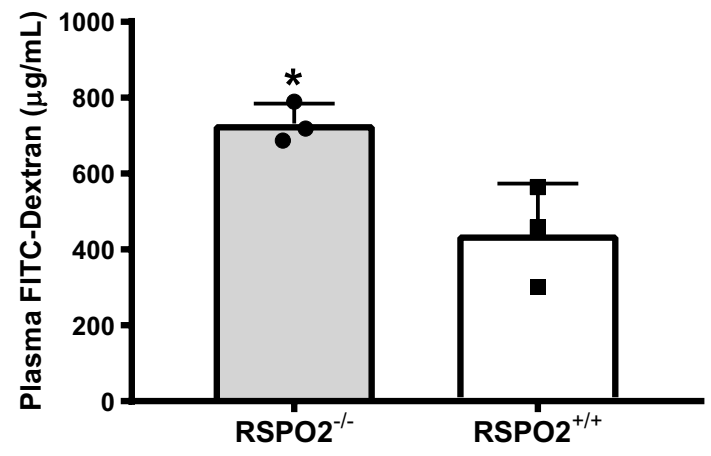

b

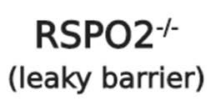

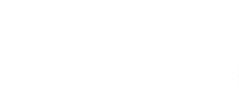

;
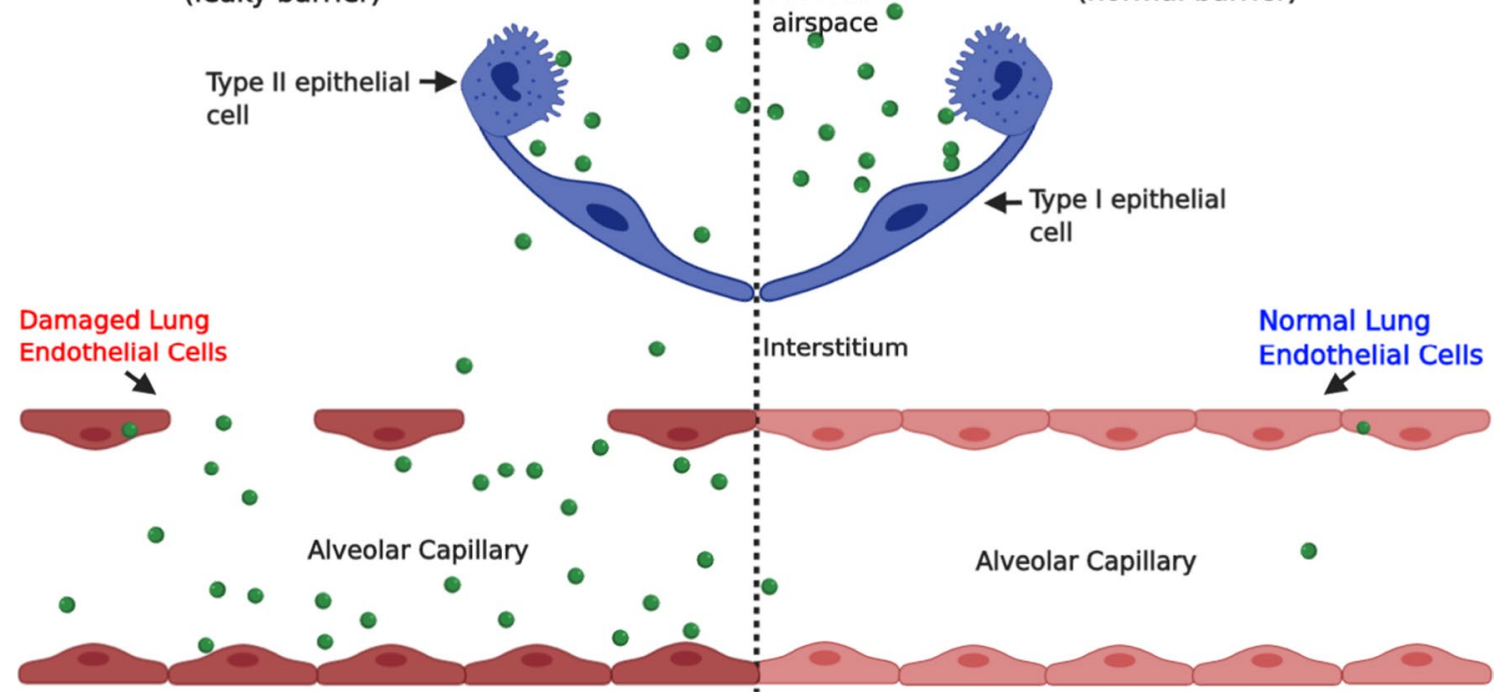

- FITC-Dextran Molecule

Fig. 3 RSPO2 deletion increases lung permeability, as determined by a FITC-dextran assay. a A significant increase in average plasma dextran concentration $\left(\mathrm{ug} / \mathrm{ml}\right.$ ) was observed in $\mathrm{RSPO}^{-/-}$mice compared to identically treated $\mathrm{RSPO} 2^{+/+}$mice $48 \mathrm{~h}$ after TM administration. $\mathbf{b}$ The movement of FITC-Dextran into normal alveolar capillaries (right side) and damaged alveolar capillaries (left side) during a murine model of endothelial cell barrier damage. Shown are the two crucial cell barriers, epithelial cells and endothelial cells. After intranasal instillation of FITC-Dextran solution, FITC-labeled molecules travel through the interalveolar space and interstitium into alveolar capillaries. Each dot represents the average of each experimental group in each of $n=3$ independent experiments. Statistical significance was demonstrated when mice from the control group and experimental group were averaged within each of the independent experiments to control for the inherent variability in FITC-Dextran administration. When individual mice were pooled regardless of experiment, the results show a very similar trend as the averaged group, though not statistically significant. ${ }^{*}=$ A P value of less than 0.05 was considered significant

\section{Limitations}

Since we utilized a global UBC-CreERT2 model to delete RSPO2, our studies cannot identify the most relevant cellular producers of RSPO2. Moreover, RSPO2 itself is a secreted, diffusible factor. As such, it is difficult to know either the cellular source of RSPO2 or the cell types(s) responding to RSPO2 signals. Future studies should use careful lineage-specific deletion to address these limitations.

\section{Abbreviations}

4-OHT: 4-Hydroxytamoxifen; ARDS: Acute respiratory distress syndrome; BALF: Bronchoalveolar lavage fluid; DMEM: Dulbecco's modified Eagle's medium; DMSO: Dimethyl sulfoxide; FITC: Fluorescein isothiocyanate; ICC: 
Immunocytochemical; LGRs: Leucine-rich repeat-containing G-protein coupled receptors; MPO: Myeloperoxidase; P/S: Penicillin-streptomycin; qPCR: Quantitative PCR; RSPO2: R-spondin 2; $\mathrm{RSPO}^{-1-}$ : R-spondin 2-deficient; $\mathrm{RSPO}^{+/+}$: R-spondin 2 control; TM: Tamoxifen; UBC-CreERT2: Inducible Cre.

\section{Acknowledgements}

We thank Katherine M. Neupauer, Chetan Rane, Joseph N. Farschian, Yasaman Moharrer, Horace M. Delisser, Kotaro Sasaki, and Stephanie Adams for their assistance in performing these studies. We acknowledge biorender.com for their free public domain vector lung and molecule illustrations used in this manuscript. A. E. Vaughan supervised this work.

\section{Authors' contributions}

Conception and design: SRJ, MFDMC, and AEV; data acquisition: SRJ, MFDMC, AIW, SA, GP, KQ, and CFP; data analysis: SRJ, MFDMC, CFP, GZ, and AEV; review and edit manuscript: SRJ, MFDMC, KH, DH, and AEV. All authors read and approved the final manuscript.

\section{Funding}

This work was supported by NIH Grant Numbers R00HL131817, U01-Al125940, and Al095289. The content is solely the responsibility of the authors and does not necessarily represent the official views of the National Institutes of Health. The funding body, $\mathrm{NIH}$, did not play a role in the design of the study, the collection, analysis, and interpretation of data, or in writing the manuscript.

\section{Availability of data and materials}

All data generated or analyzed during this study is included in this published article. Accompanying unprocessed, raw data is available from the corresponding author on reasonable request.

\section{Ethics approval and consent to participate}

All studies were approved by the University of Pennsylvania's Institutional Animal Care and Use Committees, protocol 806262, and followed all NIH Office of Laboratory Animal Welfare guidelines. No human/patient studies were performed.

\section{Consent for publication}

No human studies were performed, so not applicable.

\section{Competing interests}

The authors declare that they have no competing interests.

\section{Author details}

${ }^{1}$ Department of Biomedical Sciences, School of Veterinary Medicine, University of Pennsylvania, 3800 Spruce St., Old Vet 372E, Philadelphia, PA 19104, USA. ${ }^{2}$ Institute for Regenerative Medicine, University of Pennsylvania, Philadelphia, PA 19104, USA. ${ }^{3}$ Department of Orthopaedic Surgery, University of Michigan School of Medicine, Ann Arbor, MI 48109, USA.

Received: 2 December 2019 Accepted: 28 January 2020 Published online: 04 February 2020

\section{References}

1. de Lau WBM, Snel B, Clevers HC. The R-spondin protein family. Genome Biol. 2012;13:242.

2. Bell SM, Schreiner CM, Wert SE, Mucenski ML, Scott WJ, Whitsett JA. R-spondin 2 is required for normal laryngeal-tracheal, lung and limb morphogenesis. Development. 2008;135:1049-58.

3. Munguía-Reyes A, Balderas-Martínez YI, Becerril C, Checa M, Ramírez R, Ortiz B, et al. R-spondin-2 is upregulated in idiopathic pulmonary fibrosis and affects fibroblast behavior. Am J Respir Cell Mol Biol. 2018;59:65-76.

4. Wu L, Zhang W, Qian J, Wu J, Jiang L, Ling C. R-spondin family members as novel biomarkers and prognostic factors in lung cancer. Oncol Lett. 2019;18:4008-15.
5. Grommes J, Soehnlein O. Contribution of neutrophils to acute lung injury. Mol Med. 2011;17:293-307.

6. Kobayashi Y. The role of chemokines in neutrophil biology. Front Biosci. 2008;13:2400-7.

7. Laval J, Ralhan A, Hartl D. Neutrophils in cystic fibrosis. Biol Chem. 2016;397:485-96.

8. Kirsebom FCM, Kausar F, Nuriev R, Makris S, Johansson C. Neutrophil recruitment and activation are differentially dependent on MyD88/ TRIF and MAVS signaling during RSV infection. Mucosal Immunol. 2019;12(5):1244-55. https://doi.org/10.1038/s41385-019-0190-0.

9. Hsia CCW, Hyde DM, Weibel ER. Lung structure and the intrinsic challenges of gas exchange. Compr Physiol. 2016;6:827-95.

10. Mathew B, Lakshminrusimha S. Noninvasive monitoring of gas exchange. In: Assisted ventilation of the neonate: an evidence-based approach to newborn respiratory care. 6th ed. 2017. p. 97-107.e102.

11. Spadaro S, Park M, Turrini C, Tunstall T, Thwaites R, Mauri T, et al. Biomarkers for acute respiratory distress syndrome and prospects for personalised medicine. J Inflamm. 2019:16:1.

12. Dudley AC. Tumor endothelial cells. Cold Spring Harb Perspect Med. 2012;2:a006536.

13. Maas SL, Soehnlein O, Viola JR. Organ-specific mechanisms of transendothelial neutrophil migration in the lung, liver, kidney, and aorta. Front Immunol. 2018;9:2739.

14. Ruzankina Y, Pinzon-Guzman C, Asare A, Ong T, Pontano L, Cotsarelis $\mathrm{G}$, et al. Deletion of the developmentally essential gene ATR in adult mice leads to age-related phenotypes and stem cell loss. Cell Stem Cell. 2007:1:113-26.

15. Vaughan AE, Brumwell AN, Chapman HA. Lung Epithelial Cell Prep. Protoc Exch. 2015. https://doi.org/10.1038/protex.2015.017.

16. Rane CK, Jackson SR, Pastore CF, Zhao G, Weiner Al, Patel NN, et al. Development of solitary chemosensory cells in the distal lung after severe influenza injury. Am J Physiol Lung Cell Mol Physiol. 2019;316:L1141-9.

17. Cummings Steven M. Fluorescein isothiocyanate (FITC) labelled dextran as a peripheral marker of lung inflammation. C35 Insights into lung disease using novel methods. American Thoracic Society; 2011. p. A4376.

18. Chen H, Wu S, Lu R, Zhang YG, Zheng Y, Sun J. Pulmonary permeability assessed by fluorescent-labeled dextran instilled intranasally into mice with LPS-induced acute lung injury. PLoS ONE. 2014;9:e101925.

19. Lau D, Mollnau H, Eiserich JP, Freeman BA, Daiber A, Gehling UM, et al. Myeloperoxidase mediates neutrophil activation by association with CD11b/CD18 integrins. Proc Natl Acad Sci USA. 2005;102:431-6.

20. Jung YS, Lee HY, Kim SD, Park JS, Kim JK, Suh PG, et al. Wnt5a stimulates chemotactic migration and chemokine production in human neutrophils. Exp Mol Med. 2013:45:e27.

21. Kannan L, Kis-Toth K, Yoshiya K, Thai TH, Sehrawat S, Mayadas TN, et al. R-spondin3 prevents mesenteric ischemia/reperfusioninduced tissue damage by tightening endothelium and preventing vascular leakage. Proc Natl Acad Sci USA. 2013;1 10:14348-53.

22. Miserocchi G, Passi A, Negrini D, Del Fabbro M, De Luca G. Pulmonary interstitial pressure and tissue matrix structure in acute hypoxia. Am J Physiol Lung Cell Mol Physiol. 2001;280:L881-7.

23. Miserocchi G, Poskurica BH, Del Fabbro M. Pulmonary interstitial pressure in anesthetized paralyzed newborn rabbits. J Appl Physiol. 1994;77:2260-8.

24. Bourque SL, Davidge ST, Adams MA. The interaction between endothelin-1 and nitric oxide in the vasculature: new perspectives. Am J Physiol Regul Integr Comp Physiol. 2011;300:R1288-95.

25. Jin YR, Turcotte TJ, Crocker AL, Han XH, Yoon JK. The canonical Wnt signaling activator, R-spondin2, regulates craniofacial patterning and morphogenesis within the branchial arch through ectodermal-mesenchymal interaction. Dev Biol. 2011;352:1-3.

\section{Publisher's Note}

Springer Nature remains neutral with regard to jurisdictional claims in published maps and institutional affiliations. 